\title{
Energy deposition around swift proton tracks in polymethylmethacrylate: How much and how far
}

\author{
Maurizio Dapor* \\ European Centre for Theoretical Studies in Nuclear Physics and Related Areas (ECT*), Bruno Kessler Foundation, \\ and Trento Institute for Fundamental Physics and Applications (INFN-TIFPA), I-38123 Trento, Italy \\ Isabel Abril ${ }^{\dagger}$ \\ Departament de Física Aplicada, Universitat d'Alacant, E-03080 Alacant, Spain
}

Pablo de Vera ${ }^{\ddagger}$

School of Mathematics and Physics, Queen's University Belfast, BT7 1NN Belfast, Northern Ireland, United Kingdom

Rafael Garcia-Molina ${ }^{\S}$

Departamento de Física - Centro de Investigación en Óptica y Nanofísica, Regional Campus of International Excellence "Campus Mare Nostrum”, Universidad de Murcia, E-30100 Murcia, Spain

(Received 10 April 2017; revised manuscript received 2 August 2017; published 22 August 2017)

\begin{abstract}
The use of proton beams in several modern technologies to probe or modify the properties of materials, such as proton beam lithography or ion beam cancer therapy, requires us to accurately know the extent to which the energy lost by the swift projectiles in the medium is redistributed radially around their tracks, since this determines several endpoints, such as the resolution of imaging or manufacturing techniques, or even the biological outcomes of radiotherapy. In this paper, the radial distribution of the energy deposited around swift-proton tracks in polymethylmethacrylate (PMMA) by the transport of secondary electrons is obtained by means of a detailed Monte Carlo simulation. The initial energy and angular distributions of the secondary electrons generated by proton impact, as well as the electronic cross sections for the ejection of these electrons, are reliably calculated in the framework of the dielectric formalism, where a realistic electronic excitation spectrum of PMMA is accounted for. The cascade of all secondary electrons generated in PMMA is simulated taking into account the main interactions that occur between these electrons and the condensed phase target. After analyzing the influence that several angular distributions of the electrons generated by the proton beam have on the resulting radial profiles of deposited energy, we conclude that the widely used Rudd and Kim formula should be replaced by the simpler isotropic angular distribution, which leads to radial energy distributions comparable to the ones obtained from more realistic angular distributions. By studying the dependence of the radial dose on the proton energy we recommend lower proton energies than previously published for reducing proximity effects around a proton track. The obtained results are of relevance for assessing the resolution limits of proton beam based imaging and manufacturing techniques.
\end{abstract}

DOI: 10.1103/PhysRevB.96.064113

\section{INTRODUCTION}

The interaction of energetic charged particles with matter is widely used both for analyzing and modifying the properties of matter. The former category includes analytical techniques such as electron microscopy, electron energy-loss spectroscopy (EELS), or Rutherford backscattering (RBS) [1-3]. Among fabrication techniques we can find ion implantation and ion or electron beam lithography, which are extensively used in the microelectronics industry [4,5]. In this context, the new technical developments in the production of focused ion beams (especially of nanometric spot size) are revolutionizing all these techniques, with the emergence of new methods that exploit the large energy loss rates and almost negligible angular deflection of energetic ions, such as scanning-ion-transmission microscopy [6], proton

\footnotetext{
*dapor@ectstar.eu

†ias@ua.es

${ }^{\ddagger}$ p.devera@qub.ac.uk

${ }^{\S}$ Corresponding author: rgm@um.es
}

beam writing [7], and ion beam lithography [8], all of them characterized by improved resolutions as compared to photon beam techniques, down to the nanometer scale.

Despite this, the applications of charged particle beams are not limited to inorganic materials. In a biomedical context, organic targets can be damaged by particle radiation, which leads to cell death or mutation. Therefore, physical mechanisms of biodamage are of great importance for radiotherapy (e.g., proton beam cancer therapy [9]) and radiation protection [10]. On top of that, proton beams are opening very exciting possibilities on the high-resolution visualization (nanometer scale) of cellular samples [11].

The capabilities of modifying materials by means of ion beams are due to the large energy lost by the particles per unit path length when moving through condensed matter targets. For swift incident projectiles (ions with energies larger than $100 \mathrm{keV} / \mathrm{u}$ and electrons above $\sim 20 \mathrm{eV}$ ), the main channel of energy loss is through inelastic collisions with the target electrons [1], which results in the production of large numbers of secondary electrons. The energy distribution of these electrons is peaked at very low energies, with most of them being ejected with a few tens of eV [12-14], so they 
can typically travel only a few nanometers. Thus, the large energies lost by the ions are redistributed in narrow distances around their paths, giving place to radial dose distributions whose sharpness depend on the characteristics (energy and nature) of the ion beam, as well as on the target nature.

The organic polymer polymethylmethacrylate (PMMA) is a very relevant target, being commonly used as a surrogate of biological tissues [15] as well as a resist for ion or electron beam lithography [16], which has motivated previous studies of the radial energy distribution at the nanometer scale in this target [17].

The radial doses that build up around the tracks of energetic ions can be adequately assessed through analytical methods $[18,19]$ or by Monte Carlo simulations [17,20,21]. While the former are faster and more efficient methods, the complete cascade of secondary and tertiary electrons (produced by the primary ion and the secondary electrons, respectively), including all the possible interaction mechanisms and complex geometries, can only be followed in detail in event-by-event Monte Carlo simulations. However, the accuracy of the results provided by the different simulation codes depends on the availability of reliable interaction probabilities (through the corresponding cross sections). The initial energy and angular spectra of the electrons ejected by the ion impact determines the initial conditions for the track structure, and to follow in detail the transport and energy deposition of these secondary electrons through the target it is required to accurately know the elastic and inelastic (including electronic excitation and ionization) inverse mean free paths of electrons in the condensed phase targets. Moreover, other interactions between the electrons and the condensed state target should also be accounted for.

Several methodologies have been used to obtain energy and angular distributions of secondary electrons produced by ion impact, going from very simple and fast semiclassical models to complex and sophisticated time-consuming $a b$ initio methods [22,23]. However, they are usually limited to particular projectile-target combinations (commonly gas phase targets), to certain ranges of energies, or they fail to provide both the energy and angular distributions of the electrons. Also, these methods have to be complemented with models for electronic excitation, which are scarcer. This is the case, for example, of the recent study by Udalagama et al. [17]. While implementing the promising Hansen-KocbachStolterfoht method for ionization in their Monte Carlo code, these researchers have to complement it with different models and databases for electronic interactions of ions and electrons. Alternatively, a model based on the dielectric formalism $[24,25]$ was recently proposed $[13,26]$ to obtain reliable energy and angular distributions of electrons generated by energetic projectiles in organic targets, such as PMMA. This method has the advantage of being computationally efficient, while it allows us to obtain, within a unique framework, both the probabilities of electronic excitation and ionization, including the total energy loss and the energy and angular distributions of secondary electrons, both for the impact of ion and electron beams.

In this paper we present detailed simulations of the radial dose distribution around the trajectory of swift protons $(0.1-$ $10 \mathrm{MeV}$ ) moving through PMMA, by using the event-by- event Monte Carlo code SEED (Secondary Electron Energy Distribution) [27]. This code implements the interactions of electrons in PMMA based on the realistic initial distribution of secondary electrons generated along the proton's path, according to the new model based on the dielectric response function formalism (DRF) $[13,26,28]$, which provides the total number of generated electrons, as well as their initial energy and angular distributions. Besides inelastic (excitation and ionization) interactions, the code also accounts for additional relevant interactions, namely, Coulomb scattering, as well as other features of the condensed phase, i.e., electron-phonon interaction, and trapping phenomena due to the polaronic effect, which are usually missing in other Monte Carlo codes.

The extent to which the energy lost by the protons is distributed around their path is studied as a function of the proton energy and compared to the recent simulations by Udalagama et al. [17]. Some trends of the dependence of the radial dose on the initial proton energy are identified, which are relevant to determine the resolution of proton beam-based technologies. Also, the effect of considering realistic, instead of more simplified, angular distributions of ejected electrons is discussed. This leads to some useful recommendations on how to account in a simple manner for this relevant initial stage in electron transport simulations.

The paper is organized as follows. In Sec. II we feature the dielectric formalism methodology to obtain electronic excitation and ionization cross sections, paying special attention to the calculation of the energy and angular spectra of secondary electrons generated by swift proton impact in condensed phase targets. The main details and recent development of the event-by-event Monte Carlo code SEED are explained in Sec. III. The electronic cross sections of the generated electrons are used in the SEED code in Sec. IV, where we report our results on the radial energy deposition profiles produced by swift protons in PMMA. The final remarks and conclusions are presented in Sec. V.

\section{INTERACTIONS OF CHARGED PARTICLES AND GENERATION OF SECONDARY ELECTRONS}

The main energy loss channel of the swift charged particles in the energy range studied in this paper is through interactions with the electrons of the target, which can result in electronic excitations and, much more often, in ionizations, leading to the production of secondary electrons. The dielectric formalism $[24,25]$ provides a suitable framework to calculate all the necessary electronic cross sections (for the total energy loss, as well as for the energy and angular distributions) of the electrons generated and moving in condensed phase targets.

\section{A. Proton projectiles}

Let us consider a swift proton with mass $M$ and atomic number $Z$ moving with kinetic energy $T$ through a medium characterized by its dielectric function $\varepsilon(k, \omega)$. The doubly differential inverse mean free path (DDiMFP) [29] for inelastic interactions with energy transfer $\hbar \omega$ and momentum transfer $\hbar k$ is given by [30]:

$$
\frac{d^{2} \Lambda_{\mathrm{H}}^{\mathrm{excit}-\mathrm{ioniz}}(T)}{d \omega d k}=\frac{e^{2} Z^{2} M}{\pi \hbar T} \frac{1}{k} \operatorname{Im}\left[\frac{-1}{\varepsilon(k, \omega)}\right],
$$


where $e$ is the fundamental charge and $\operatorname{Im}[-1 / \varepsilon(k, \omega)]$ is the energy loss function (ELF) of the target, which is a key element of this methodology, since it contains the relevant information on the energy $\hbar \omega$ and momentum $\hbar k$ electronic excitation spectrum of the material. The above expression is very useful, because it allows, besides the calculation of the integrated energy loss quantities (projectile energy loss and number of electrons produced per unit path length), the determination of the energy and angular distribution of secondary electrons, when a relation between the energy $\hbar \omega$ (and the momentum $\hbar k$ ) transferred by the proton and the energy $W$ of the secondary electrons (and their ejection angle $\theta$ ) is provided.

Integration of Eq. (1) over the whole energy and momentum plane (the Bethe surface) gives the total inverse mean free path (iMFP, hereafter) for inelastic interactions:

$$
\Lambda_{\mathrm{H}}^{\text {excit-ioniz }}(T)=\frac{e^{2} Z^{2} M}{\pi \hbar T} \int_{0}^{\omega_{\max }} d \omega \int_{k_{1}}^{k_{2}} \frac{d k}{k} \operatorname{Im}\left[\frac{-1}{\varepsilon(k, \omega)}\right] .
$$

Due to momentum and energy conservation, the integration limits are $\hbar k_{1,2}=\sqrt{2 M}(\sqrt{T} \mp \sqrt{T-\hbar \omega})$ and $\hbar \omega_{\max } \simeq$ $4 m T / M$ [31], with $m$ being the electron mass. The iMFP is directly related to the microscopic cross section $\sigma$ by $\Lambda=\mathcal{N} \sigma$, where $\mathcal{N}$ is the target atomic or molecular density. Equation (2) includes both electronic excitations and ionizations. In the dielectric response function formalism (DRF) [13] it was shown that it is possible to introduce a mean binding energy $\bar{B}$ for the outer shell electrons, in such a way that secondary electrons will be ejected, when $\hbar \omega>\bar{B}$, with kinetic energy $W=\hbar \omega-\bar{B}$. For inner shells, this relation reads $W=\hbar \omega-B_{j, n l}$, where $B_{j, n l}$ is the ionization energy of the $(n, l)$ subshell of the $j$ th atomic constituent of a compound target. Following this reasoning, the singly differential inverse mean free path (SDiMFP) for ionization is given by:

$$
\begin{aligned}
\frac{d \Lambda_{\mathrm{H}}^{\mathrm{ioniz}}(T, W)}{d W}= & \frac{e^{2} Z^{2} M}{\pi \hbar^{2} T} \int_{\kappa_{1}}^{\kappa_{2}} \frac{d k}{k} \operatorname{Im}\left[\frac{-1}{\varepsilon(k, W+\bar{B})}\right]_{\text {outer }} \\
& +\frac{e^{2} Z^{2} M}{\pi \hbar^{2} T} \sum_{j} v_{j} \sum_{n l} \int_{\zeta_{1}}^{\zeta_{2}} \frac{d k}{k} \\
& \times \operatorname{Im}\left[\frac{-1}{\varepsilon\left(k, W+B_{j, n l}\right)}\right],
\end{aligned}
$$

where we have split the ELF into two contributions, namely $\operatorname{Im}[-1 / \varepsilon(k, W+\bar{B})]_{\text {outer }}$ corresponding to the outer shell electrons and $\operatorname{Im}\left[-1 / \varepsilon\left(k, W+B_{j, n l}\right)\right]$ for the inner shells. Here $v_{j}$ is the stoichiometric coefficient of each atomic constituent $j$ of the target. The integration limits are given by $\hbar \kappa_{1,2}=\sqrt{2 M}(\sqrt{T} \mp \sqrt{T-W-\bar{B}})$ and $\hbar \zeta_{1,2}=$ $\sqrt{2 M}\left(\sqrt{T} \mp \sqrt{T-W-B_{j, n l}}\right)$. In order to take into account the condensed phase state of the target, its ELF over the whole energy and momentum transfer domain is obtained by means of the MELF-GOS (Mermin Energy Loss-Generalized Oscillator Strengths) methodology [32,33], where the contribution from the outer-shell electron excitations is separated from the contribution due to the $(n, l)$-inner-shell electron ionizations of each atom $j$ of the target. The former is described by means of a weighted sum of Mermin-type ELF [34], while the latter is accounted for by hydrogenic generalized oscillator strengths (GOS) [2]. The MELF-GOS model only needs suitable (experimental or theoretical) optical ELF as input data and then extends them over the whole momentum plane by virtue of the analytical form of the Mermin dielectric function and the hydrogenic GOS. Therefore, many-body interactions as well as physical-state effects are automatically included in this treatment. The energy loss function obtained by the MELF-GOS method has been shown to properly reproduce the experimental Bethe surface of liquid water [35] and other materials [36,37], and it accurately satisfies a set of physical constrains [38].

We will focus our study on the case of protons incident on PMMA, a polymer commonly used as a tissue-equivalent material as well as a lithographic resist, whose ELF in the whole $(\hbar \omega, \hbar k)$ domain was obtained [28] from the experimental optical ELF [39]. For this material, the mean binding energy $\bar{B}$ is the only (a priori) undetermined parameter in our model. Although $20 \mathrm{eV}$ was considered previously as a good approximation of $\bar{B}$ for organic materials when there are no available data [14] (which agrees with the weighted average of the first ionization energies of the atomic constituents of PMMA), in what follows we will use $\bar{B}=15 \mathrm{eV}$, a value in between the previous one and $12 \mathrm{eV}$, which is the weighted average of the lowest electronic energy levels of a model molecule for PMMA [39].

Figure 1 shows the initial energy distribution of the electrons generated by $0.1-10 \mathrm{MeV}$ protons incident on PMMA, as obtained from Eq. (3). The left panel depicts, in linear scale, the SDiMFP at low electron energies $W$, while the right panel (in logarithmic scale) corresponds to the higher electron energies. It is worth noticing that all the energy distributions are peaked at the same electron energy (around 6-8 eV), independently of the initial proton energy; these low electron energies are considered to be very important in affecting organic target properties [40]. However, the absolute value of the SDiMFP decreases (i.e., less electrons are ejected) when the proton energy $T$ increases, with the differences in the SDiMFP lowering as $T$ grows. Also, increasing the proton energy makes the tail of the SDiMFP to extend to larger secondary electron energies; this is due to the maximum energy that can be transferred to secondary electrons in inelastic collisions, which is given by $W_{\max } \simeq 4 m T / M$. The SDiMFP vanishes for each proton energy as $W$ approaches this kinematic limit. These three features (maximum of the spectrum at a position independent of the proton energy, number of electrons decreasing for increasing proton energies, and longer high energy tails for higher proton energies) will be important to understand the results presented in Sec. IV.

The angular distribution of the electrons generated by ion impact is another essential input quantity required for Monte Carlo simulations. This distribution is obtained within the DRF model by using a proportionality relation between the scattering angle $\psi$ of the incident proton and the ejection angle $\theta$ of the secondary electron, $\psi=\alpha \theta$. This relation was motivated by the resemblance of the angular distributions of scattering angles $\psi$ calculated within the DRF model and the experimental distributions of ejection angles $\theta$ of secondary electrons and is supported by the good agreement of the model with available experimental data [26]. The exact value of $\alpha$ is obtained from the binary encounter angle (BEA) as 


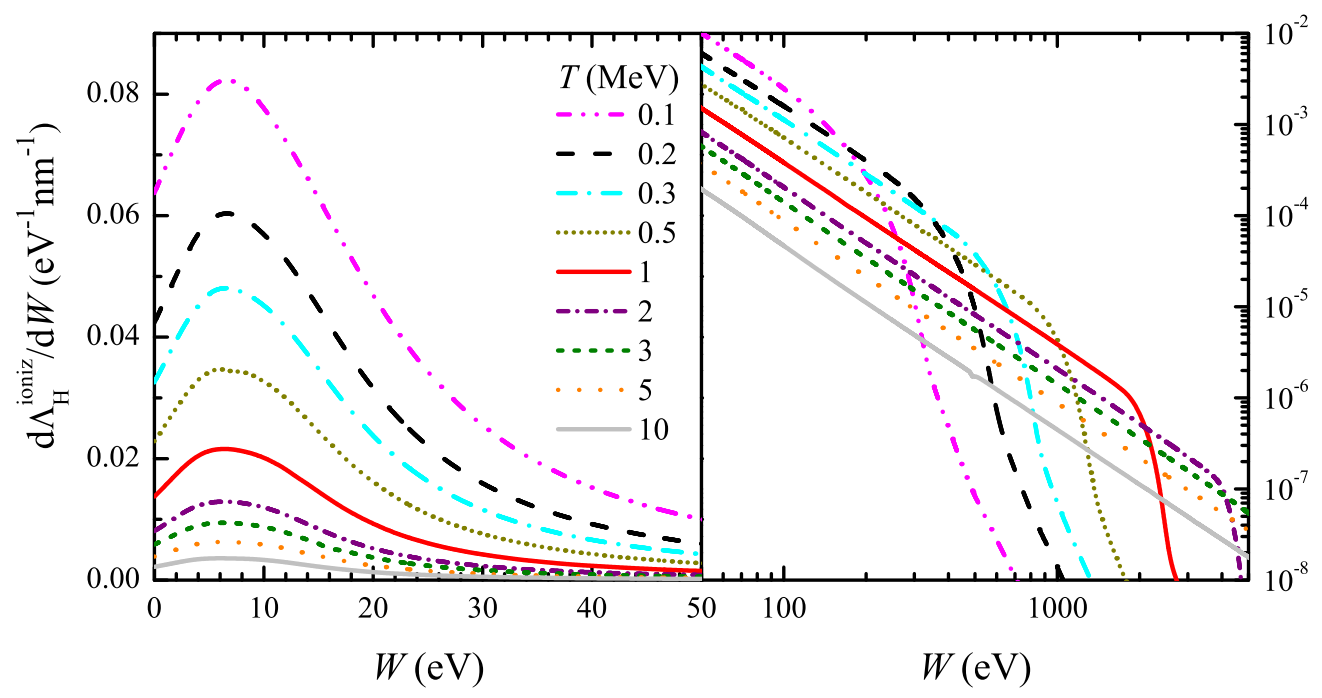

FIG. 1. Energy distribution of electrons generated by $0.1-10 \mathrm{MeV}$ proton impact in PMMA, as a function of the electron energy $W$, according to Eq. (3).

$\alpha=\psi^{\mathrm{BEA}} / \theta^{\mathrm{BEA}}$, where both the angular spectra as a function of $\psi$ and $\theta$ have a clear maximum, so they can be correlated. The rest of the ejection (and scattering) angles are obtained by the momentum distribution of the electrons in the target as given by its ELF. For further details, the reader is referred to Ref. [26].
The energy and angular doubly differential inverse mean free path (DDiMFP) of electrons emitted with energy $W$ and angle $\theta$ (per unit energy and solid angle $d \Omega=2 \pi \sin \theta d \theta$ ) due to a proton with atomic number $Z$ and mass $M$ impacting with energy $T$ on a target characterized by its $\operatorname{ELF}, \operatorname{Im}(-1 / \varepsilon)$, is given by [26]:

$$
\begin{aligned}
\frac{d^{2} \Lambda_{\mathrm{H}}^{\mathrm{ioniz}}(T, W, \theta)}{d W d \Omega}= & \frac{\alpha e^{2} Z^{2}}{2 \pi^{2} \hbar^{2} \sin \theta} \frac{M}{T} F_{\text {Salin }} \times\left\{\frac{\sqrt{T(T-(W+\bar{B}))} \sin (\alpha \theta)}{2 T-(W+\bar{B})-2 \sqrt{T(T-(W+\bar{B}))} \cos (\alpha \theta)} \operatorname{Im}\left[\frac{-1}{\varepsilon(k, W+\bar{B})}\right]_{\mathrm{outer}}\right. \\
& \left.+\sum_{j} v_{j} \sum_{n l} \frac{\sqrt{T\left(T-\left(W+B_{j, n l}\right)\right)} \sin (\alpha \theta)}{2 T-\left(W+B_{j, n l}\right)-2 \sqrt{T\left(T-\left(W+B_{j, n l}\right)\right)} \cos (\alpha \theta)} \operatorname{Im}\left[\frac{-1}{\varepsilon\left(k, W+B_{j, n l}\right)}\right]\right\}
\end{aligned}
$$

In this equation, the Salin's factor $F_{\text {Salin }}$ accounts for two center effects that attract the ejected electron to the projectile's field after the collision, with electron capture to the continuum [41].

By definition, the dielectric formalism (based on the first Born or plane wave approximation) cannot account for two center effects. The incident projectile and the ejected electron are described by plane waves and hence do not obey the correct boundary conditions, i.e., the electron wave being distorted by the field of the projectile both before and after the collision. More accurate methodologies exist to account for these two center effects, mainly represented by the continuum distorted wave (CDW) approximation, where these boundary conditions are correctly considered $[42,43]$. Within the first Born approximation, it is customary to multiply the cross sections by the Salin's factor (related to the reciprocal of the absolute value of the difference between the proton and electron velocity vectors), in the form of a semiempirical factor that corrects the cross section for the electron capture to the continuum $[22,44]$.

Analogously to Eq. (3), in Eq. (4) there are contributions due to the outer-shell electrons (first term in the r.h.s.) and from the inner shells of each type of atom in a compound target (second term in the r.h.s). We show in Fig. 2 the angular distribution of the electrons generated by the impact of $1 \mathrm{MeV}$ protons in PMMA, for electron energies $W$ in the range from $10 \mathrm{eV}$ to $2100 \mathrm{eV}$; to our knowledge no experimental data are available to compare with. Figure 2 shows that electrons emitted at low energies $W$ have a rather isotropic angular distribution, which develops a structure when $W$ increases, whose maximum value coincides with the angle $\theta$ corresponding to the binary encounter peak.

The increase of the curves at $\theta \rightarrow 180^{\circ}$ is just an artifact coming from the term $\sin \theta$ in the denominator of Eq. (4) when the DDiMFP is plotted per unit solid angle. The observed increase in the cross section for $W=500 \mathrm{eV}$ at $\theta \leqslant 30^{\circ}$ results from electron capture to the continuum, which is accounted for by the Salin's factor. At $W=500 \mathrm{eV}$, the ejected electrons have a speed close to that of the $1 \mathrm{MeV}$ proton, so they are preferentially ejected in the forward direction attracted by the field of the proton.

\section{B. Electron projectiles}

As a consequence of the energy delivered by a swift proton along its path, secondary electrons are emitted due to ionization of the target atoms. These electrons move away from the 


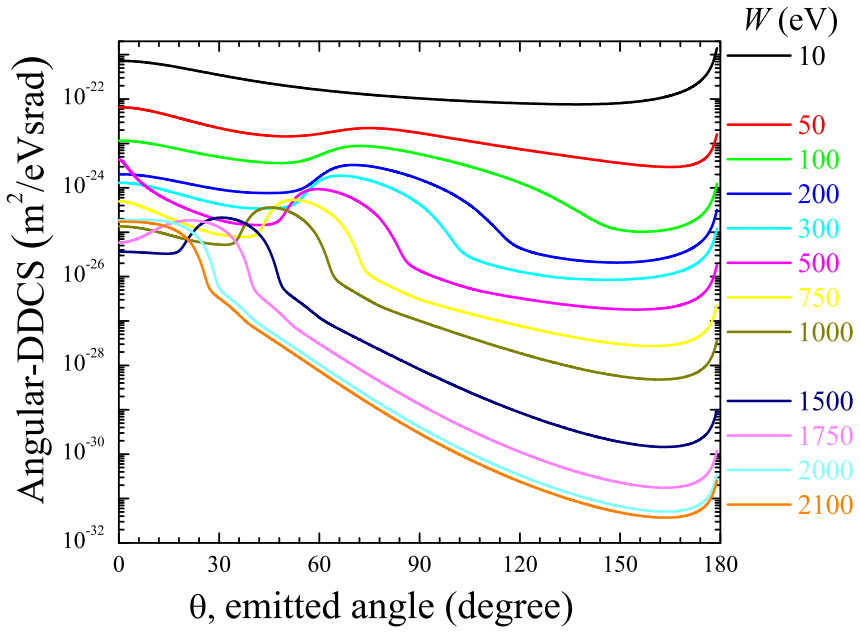

FIG. 2. Angular distribution $d^{2} \Lambda_{\mathrm{H}}^{\text {ioniz }} / d W d \Omega$ of secondary electrons generated by the impact of a $1 \mathrm{MeV}$ proton beam incident on PMMA, as a function of their emitted angle $\theta$, for several electron energies $W$.

region where they originated, experiencing elastic and inelastic scattering with the target constituents, losing energy through excitations and producing new ionizations, which results in an avalanche of electrons.

To simulate the distribution of energy carried by these electrons we use the SEED code, whose details are described elsewhere [27]. This is an event-by-event Monte Carlo code that has been previously applied to simulate the energy distribution of the secondary electrons generated along ion tracks [21] and by electron irradiation of solid targets [45].

The inverse mean free path $\Lambda_{\mathrm{e}}^{\text {elast }}$ for the elastic collisions suffered by electrons is calculated using the Mott theory [46], for which the Dirac equation is numerically solved in a central field. This procedure, known as the "relativistic partial wave expansion method," has been demonstrated to provide excellent results when compared to experimental data
$[47,48]$ and is the standard procedure to obtain elastic iMFP for electrons. However, it should be kept in mind that due to the electron energies involved in this work, no relativistic corrections are required in the electron kinematics.

Concerning the inelastic scattering of the electrons, for electron energies higher than $\sim 20-50 \mathrm{eV}$, two main interaction channels have to be considered: ionization and excitations.

The SDiMFP for the ionizations produced by energetic electrons is calculated by using the DRF model presented in Eq. (3) but now taking into account the specific nature of the electron projectile. To proceed in this way the electron inelastic scattering must consider Pauli's exclusion principle, the indistinguishability between the incident and the struck electron, as well as the exchange effects [49]. It is commonly accepted that after the collision the most energetic electron is the primary one, which implies that the maximum energy transferred by an electron with energy $T$ cannot exceed the value $\hbar \omega_{\max }^{\text {ioniz }}=\left(T+E_{\text {binding }}\right) / 2$, where $E_{\text {binding }}=$ $\bar{B}$ for the outer-shell electron excitations and $E_{\text {binding }}=B_{j, n l}$ for the $(n, l)$ inner-shell electron excitation of the atom $j$ of the target. Besides, due to Pauli's exclusion principle, the primary electron after the collision cannot lie on a target occupied level, so $\hbar \omega_{\max }^{\text {ioniz }}=T-E_{\text {Pauli }}$, where $E_{\text {Pauli }}$ is the Fermi energy for metals, or a value close to a few eV for semiconductors and insulators [49], although the exact value of $E_{\text {Pauli }}$ does not change sensibly the electron SDiMFP.

According to the previous discussion, the upper limit of the energy transfer in an inelastic collision between electrons (leading to ionization) is $\hbar \omega_{\max }^{\text {ioniz }}=\min \left[\left(T+E_{\text {binding }}\right) / 2, T-\right.$ $\left.E_{\text {Pauli }}\right]$. Additional corrections to the dielectric formalism are necessary to correctly describe very low energy electrons, so an exchange correction term $f_{\text {exch }}$ has to be included in the model. We have used the Ochkur exchange correction term [50], $f_{\text {exch }}(k)=1-\left(\hbar^{2} k^{2}\right) /(2 m T)+\left[\left(\hbar^{2} k^{2}\right) /(2 m T)\right]^{2}$, which comes from the leading term of an expansion of the Born-Oppenheimer amplitude in series of the electron energy $T$ [51]. Taking into account the previous considerations, the ionization SDiMFP due to the impact of a swift electron with kinetic energy $T$ is given by:

$$
\frac{d \Lambda_{\mathrm{e}}^{\text {ioniz }}(T, W)}{d W}=\frac{e^{2} m}{\pi \hbar^{2} T} \int_{\kappa_{e, 1}}^{\kappa_{e, 2}} \frac{d k}{k} f_{\text {exch }}(k) \operatorname{Im}\left[\frac{-1}{\varepsilon(k, W+\bar{B})}\right]_{\text {outer }}+\frac{e^{2} m}{\pi \hbar^{2} T} \sum_{j} v_{j} \sum_{n l} \int_{\zeta_{e, 1}}^{\zeta_{e, 2}} \frac{d k}{k} f_{\text {exch }}(k) \operatorname{Im}\left[\frac{-1}{\varepsilon\left(k, W+B_{j, n l}\right)}\right] .
$$

When the incident electron undergoes an inelastic collision, the Monte Carlo code also requires knowing whether ionization or excitation of the target electrons occurs. In order to do that the electronic iMFP should be known, which is obtained from Eq. (2) but applying the exchange correction and using the correct integration limits discussed in the previous paragraph. This can be expressed as the sum of the excitation and ionization processes: $\Lambda_{\mathrm{e}}^{\text {excit-ioniz }}=\Lambda_{\mathrm{e}}^{\text {excit }}+\Lambda_{\mathrm{e}}^{\text {ioniz }}$. As done previously, we use the mean binding energy to separate excitation from ionization. Proceeding in this manner, and according to the dielectric framework, the excitation iMFP of an electron moving with kinetic energy $T$ in a condensed target is given by:

$$
\Lambda_{\mathrm{e}}^{\mathrm{excit}}(T)=\frac{e^{2} m}{\pi \hbar T} \int_{0}^{\omega_{\max }^{\text {excit }}} d \omega \int_{\kappa_{e, 1}}^{\kappa_{e, 2}} \frac{d k}{k} \operatorname{Im}\left[\frac{-1}{\varepsilon(k, W+\bar{B})}\right]_{\text {outer }},
$$

where the maximum energy transferred by the electron when an excitation process takes place is $\hbar \omega_{\max }^{\text {excit }}=\min (\bar{B}, T-$ $\left.E_{\text {Pauli }}\right)$. On the other hand, the ionization iMFP for an electron with energy $T$ is given by the following 
expression:

$$
\begin{aligned}
\Lambda_{\mathrm{e}}^{\mathrm{ioniz}}(T)= & \frac{e^{2} m}{\pi \hbar T} \int_{\bar{B} / \hbar}^{\omega_{\mathrm{max}}^{\mathrm{ionix}}} d \omega \int_{\kappa_{e, 1}}^{\kappa_{e, 2}} \frac{d k}{k} f_{\mathrm{exch}}(k) \operatorname{Im}\left[\frac{-1}{\varepsilon(k, W+\bar{B})}\right]_{\mathrm{outer}}+\frac{e^{2} m}{\pi \hbar T} \sum_{j} v_{j} \sum_{n l} \int_{B_{j, n l / \hbar}}^{\omega_{\mathrm{max}}^{\text {ion }}} d \omega \int_{\zeta e, 1}^{\zeta_{e, 2}} \frac{d k}{k} f_{\text {exch }}(k) \\
& \times \operatorname{Im}\left[\frac{-1}{\varepsilon\left(k, W+B_{j, n l}\right)}\right] .
\end{aligned}
$$

As we discussed earlier in relation to Eq. (5), in the case of ionization it is necessary to consider the indistinguishability between the primary and the struck electrons, as well as Pauli's exclusion principle (which enters through the maximum energy transfer appearing in the integration limit) and also the exchange correction through the Ochkur term $f_{\text {exch }}$. In the case of excitation processes the indistinguishability and the exchange corrections do not apply.

We present in Fig. 3 the excitation (dashed line) and the ionization (solid line) iMFP of electrons in PMMA as a function of the incident electron energy $T$. The dotted line represents the total electronic iMFP. The results were obtained by using Eqs. (6) and (7) with the ELF of PMMA described by the MELF-GOS model [28]. We find that the maximum value of the electron ionization iMFP occurs at around $90 \mathrm{eV}$, whereas the excitation iMFP has a maximum around $18 \mathrm{eV}$.

When the electron energy $T$ becomes lower than $\sim$ $20-50 \mathrm{eV}$ it can lose energy in many small amounts interacting with phonons, in particular with the optical modes of lattice vibrations. In his theory of the electron-phonon interaction, Fröhlich [52] described the interaction of free conduction electrons with the longitudinal optical mode lattice vibrations. Since, according to Ganachaud and Mokrani [53], the dispersion relation of the longitudinal phonons can be neglected in the optical branch, one can use a single phonon frequency $\omega_{\text {phon. }}$. Using the Fröhlich theory, Llacer and Garwin [54] calculated the inelastic iMFP for electron-phonon interaction

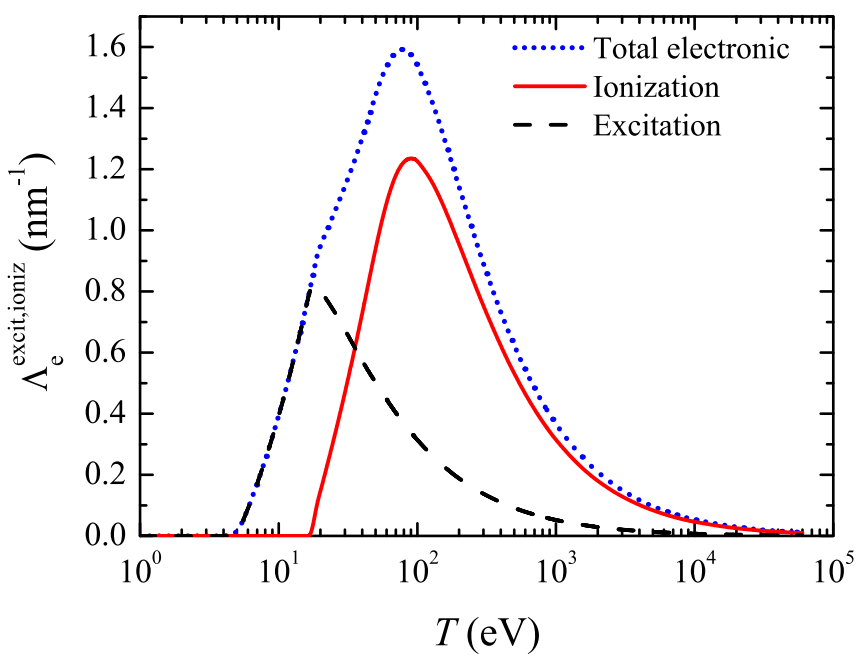

FIG. 3. Inverse mean free path (iMFP) corresponding to ionization (solid line) and excitation (dashed line) processes, as well as the total iMFP (dotted line) for electrons in PMMA, as a function of the incident electron energy $T$.
$\Lambda_{\mathrm{e}}^{\text {phon }}$ and obtained the following result:

$$
\begin{aligned}
\Lambda_{\mathrm{e}}^{\text {phon }}(T)= & \frac{1}{a_{0}}\left(\frac{n(\tau)+1}{2}\right)\left(\frac{\varepsilon(0)-\varepsilon(\infty)}{\varepsilon(0) \varepsilon(\infty)}\right) \frac{\hbar \omega_{\text {phon }}}{T} \\
& \times \ln \left[\frac{1+\sqrt{1-\hbar \omega_{\text {phon }} / T}}{1-\sqrt{1-\hbar \omega_{\text {phon }} / T}}\right],
\end{aligned}
$$

where $n(\tau)=\left(e^{\hbar \omega_{\text {phon }} / k_{\mathrm{B}} \tau}-1\right)^{-1}, a_{0}$ is the Bohr radius, $k_{\mathrm{B}}$ is the Boltzmann constant, $\varepsilon(0)$ is the static dielectric constant, $\varepsilon(\infty)$ is the high frequency dielectric constant, and $\tau$ is the absolute temperature of the target. With typical values of $\hbar \omega_{\text {phon }}$ lying in the range from 0.01 to $0.1 \mathrm{eV}$ [54], we have used $\hbar \omega_{\text {phon }}=0.1 \mathrm{eV}$ in the present simulations [45].

Low-energy electrons moving in an insulating material induce around them a polarization field, which has a stabilizing effect on the electrons that generated it. The quasiparticle describing the slow electron with its polarization field around is called polaron. According to Ganachaud and Mokrani [53], the inelastic iMFP for electron-polaron interaction $\Lambda_{\mathrm{e}}^{\mathrm{pol}}$ is given by:

$$
\Lambda_{\mathrm{e}}^{\mathrm{pol}}(T)=C e^{-\gamma T},
$$

where $C$ and $\gamma$ are constants depending on the material under investigation. For PMMA we have used the values provided by Ref. [45] as they give good agreement with the experimental data by many authors both concerning secondary and total electron yield and secondary electron energy distribution. Therefore, the inelastic iMFP, which takes into account all the above mentioned inelastic scattering processes, is given by:

$$
\Lambda_{\mathrm{e}}^{\text {inel }}(T)=\Lambda_{\mathrm{e}}^{\mathrm{excit}}(T)+\Lambda_{\mathrm{e}}^{\mathrm{ioniz}}(T)+\Lambda_{\mathrm{e}}^{\mathrm{phon}}(T)+\Lambda_{\mathrm{e}}^{\mathrm{pol}}(T) .
$$

The total iMFP, which considers both elastic and inelastic inverse mean free paths, $\Lambda_{\mathrm{e}}^{\text {elast }}(T)$ and $\Lambda_{\mathrm{e}}^{\text {inel }}(T)$, is given by:

$$
\Lambda_{\mathrm{e}}^{\text {total }}(T)=\Lambda_{\mathrm{e}}^{\text {elast }}(T)+\Lambda_{\mathrm{e}}^{\text {inel }}(T) .
$$

The initial angular distribution of the electrons generated by proton impact has been described in Sec. II A and will be further discussed in Sec. IV. On the other hand, the polar scattering angle $\psi$ along the trajectory of each electron is calculated, as already noted, according to the Mott theory for elastic scattering. In the case of inelastic collisions: (i) For electron-electron ionization collisions, the deviation angle $\psi$ of the incident electron is calculated within the classical binary collision model by [22]

$$
\sin ^{2} \psi=\frac{\Delta T}{T},
$$


TABLE I. Inelastic mean free path of protons in PMMA, for several energies $T$ of the proton beam, Eq. (2).

\begin{tabular}{lc}
\hline \hline$T(\mathrm{MeV})$ & $1 / \Lambda_{\mathrm{H}}^{\text {exit-ioniz }}(T)(\mathrm{nm})$ \\
\hline 0.1 & 0.342 \\
0.3 & 0.658 \\
0.5 & 0.952 \\
1 & 1.621 \\
2 & 2.826 \\
3 & 3.943 \\
5 & 6.033 \\
10 & 10.822 \\
\hline \hline
\end{tabular}

where $\Delta T$ is the electron energy loss and $T$ is the incident electron energy. The struck out electron (which becomes a new secondary electron belonging to the cascade) has an energy $\Delta T-\bar{B}$ and is emitted isotropically [55,56].

(ii) For electron-phonon collisions, $\psi$ is obtained by [54]

$$
\cos \psi=\left(\frac{T+T^{\prime}}{2 \sqrt{T T^{\prime}}}\right)\left(1-K^{\xi}\right)+K^{\xi},
$$

where $K=\left(T+T^{\prime}+2 \sqrt{T T^{\prime}}\right) /\left(T+T^{\prime}-2 \sqrt{T T^{\prime}}\right)$, with $T$ and $T^{\prime}$ being, respectively, the electron energies before and after the electron-phonon scattering; $\xi$ is a random number uniformly distributed in the interval $[0,1]$.

\section{ELECTRON ENERGY DEPOSITION AROUND THE PROTON TRACK}

After being generated, electron trajectories follow a stochastic process, with scattering events separated by straight paths having a distribution of step lengths that follow a Poisson-type law. Once the step length is defined, the (elastic or inelastic) nature of the scattering event, the polar and azimuthal angles, and the energy losses, are all sampled using the relevant cumulative probabilities according to the usual Monte Carlo procedures [27,47,55].

By using the SEED code [27] we follow the trajectories of all the electrons generated by the proton beam (which comprise not only the ones ejected directly but also those resulting in the subsequent electron cascades). The energy delivered by these electrons to the target due to the different inelastic events (described previously) is used to calculate the radial dose $d E / d m=d E /(\rho 2 \pi r d r d z)$, i.e., energy deposited per unit mass, in a cylindrical shell of length $d z$, inner radius $r$, and outer radius $r+d r$, around the proton track in a radial interval $d r$, as a function of the radial distance $r$ from the proton's path; $\rho=1.188 \mathrm{~g} / \mathrm{cm}^{3}$ is the mass density of the target.

As the kinetic energy $T$ of the incident proton beam changes due to energy loss along its travel through the target, the value of the cylinder length $d z$ is chosen to be the corresponding proton inelastic mean free path, $1 / \Lambda_{\mathrm{H}}^{\text {excit-ioniz }}(T)$ [57]. Table I contains the values of $1 / \Lambda_{\mathrm{H}}^{\text {excit-ioniz }}(T)$ in PMMA for a few representative energies $T$, obtained by Eq. (2).

In order to model with accuracy the energy deposition, the SEED code generates (new) secondary electrons only when an ionization event takes place, which does not occur for all inelastic electronic collisions. According to the relative probability of excitation and ionization, sometimes just an electronic excitation is produced, which results in a local energy deposition without further energy transport. The SEED code distinguishes between these two electronic inelastic events according to the corresponding inverse mean free paths, which are available through expressions (6) and (7). Figure 3 shows the relative importance of the electron ionization and excitation processes in PMMA, as a function of the kinetic energy of the primary electron.

As each secondary electron produces further secondary electrons during its travel inside the solid, to obtain quantitative results it is mandatory to follow the whole electron cascade, until the moving particles are trapped in the material or reach a threshold energy, which we have taken to be $0.1 \mathrm{eV}$. The final results we will present do not crucially depend on this quantity, provided it is in the range of tenths of $\mathrm{eV}$.

\section{RESULTS AND DISCUSSION}

In this section we present the results of the simulations performed with the SEED code, as described in Sec. III, where the initial energy and angular distributions of electrons produced by the incoming protons, as well as the electronic interactions of these electrons with the medium, have been described within the dielectric formalism as explained in Sec. II.

In the first place, let us discuss the effect that the initial conditions of the track-structure code have on the simulated radial energy distribution. In order to analyze the influence of the angular distribution of the electrons ejected by proton impact on the radial dose $d E / d m$, it has been simulated by using different expressions for the angular DDiMFP, namely, isotropic (the simplest), perpendicular to the ion's path, forward, and backward DDiMFPs, as well as angular DDiMFP obtained from the more physically motivated DRF model [26], Eq. (4), and the widely used Rudd and Kim model [22], based on the binary encounter angle. In all the cases the initial energy distribution of the electrons generated by the proton beam is given by Eq. (3) with $\bar{B}=15 \mathrm{eV}$ [13].

We show in Fig. 4 the radial dose around a $1 \mathrm{MeV}$ proton track in PMMA. We can see that the dose is practically identical for the isotropic angular distribution and for the angular distribution of the DRF model [26] presented in Sec. II A. This behavior could have been inferred from Fig. 1, which shows that low energy electrons are the most abundant, and from Fig. 2, where an approximate isotropic angular distribution emerges for electrons with energy lower than $50 \mathrm{eV}$. The radial doses obtained when using the forward and the backward angular distributions agree between them, but not with the others. Finally, the Rudd and Kim angular distribution [22], which corresponds to a delta value as a function of the initial proton energy and the emitted electron energy, gives a radial dose that practically coincides with the one obtained from the simple perpendicular distribution. From the analysis of Fig. 4, we conclude that the extensively used Rudd and Kim angular distribution [22] for the electrons generated by proton ionization should be replaced by the isotropic angular distribution, which leads to radial dose distributions comparable to the ones obtained from the more realistic angular distribution of Eq. (4). The differences among the 


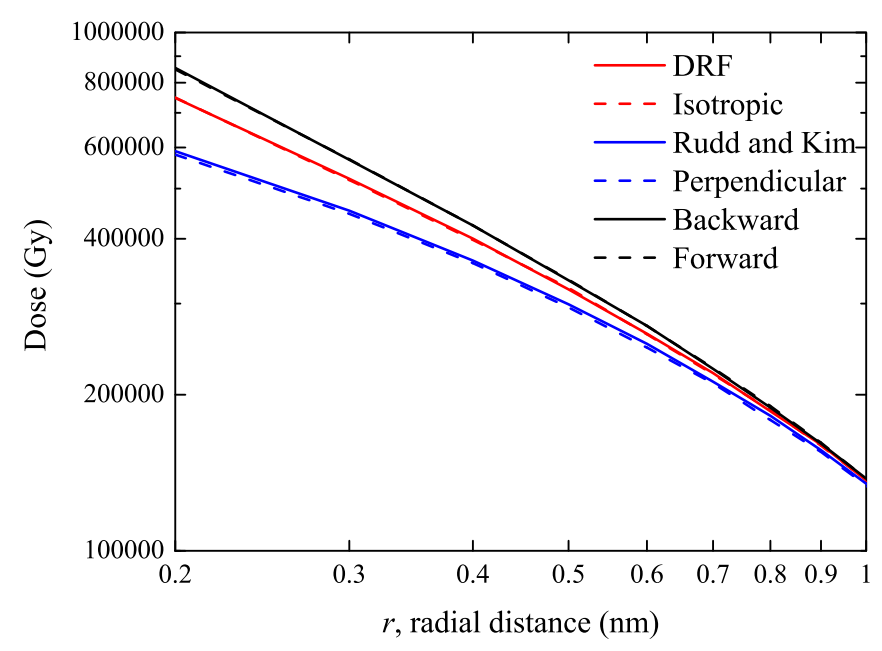

FIG. 4. SEED simulation of the radial dose, $d E / d m$, around the track of a $1 \mathrm{MeV}$ proton beam incident on PMMA, for several initial angular distributions of the electrons (indicated in the labels).

radial doses obtained from the different angular distributions disappear at distances larger than $1 \mathrm{~nm}$.

Besides the coincidences (and differences) among the radial dose distributions, it is worth noticing that the results obtained from the isotropic and DRF cases are between the (higher) backward and forward and the (lower) perpendicular and Rudd and Kim cases. In what follows, we will use the more

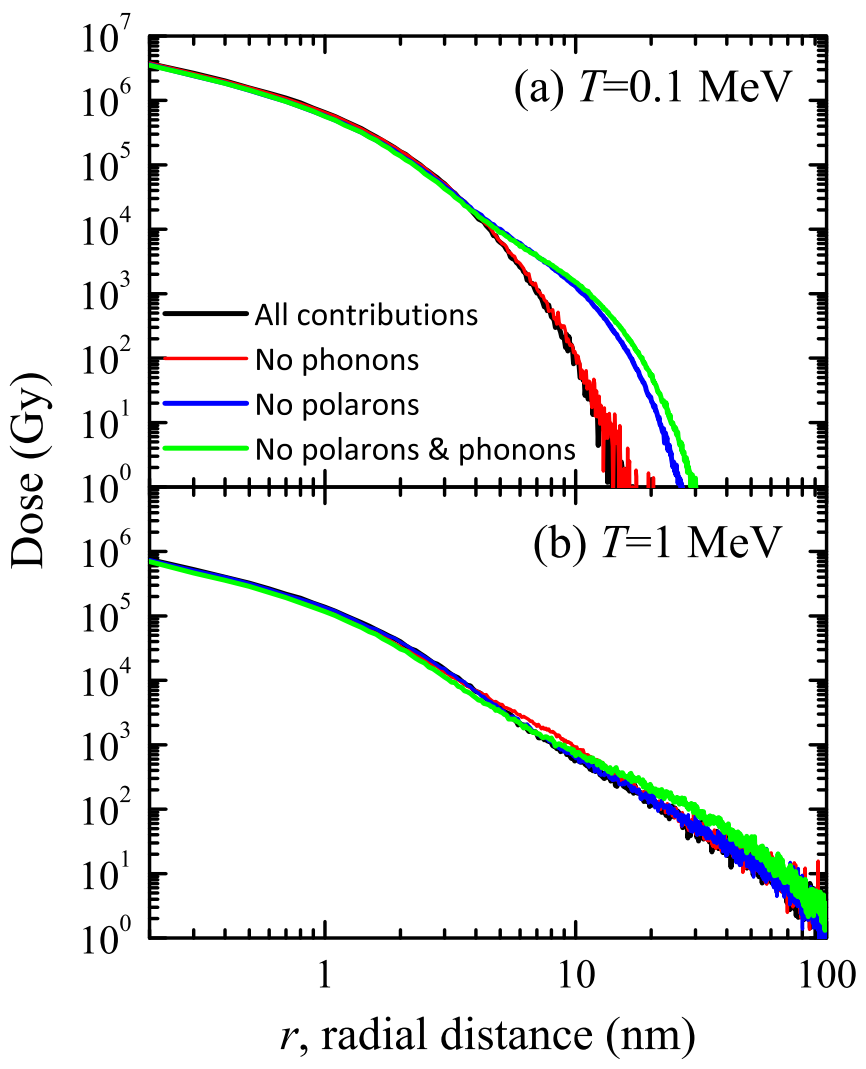

FIG. 5. Radial doses obtained when phonon and/or polaron processes are removed from the SEED simulation (as stated in the labels), for 0.1 and $1 \mathrm{MeV}$ proton impact in PMMA.

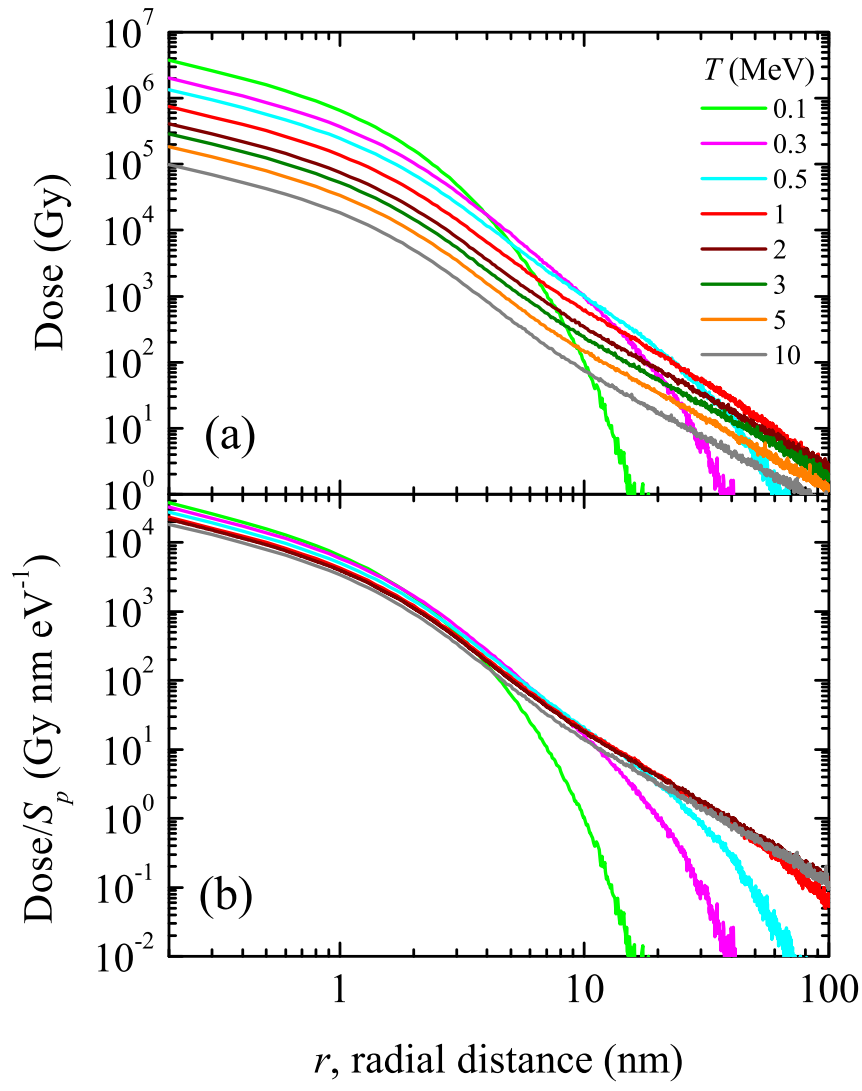

FIG. 6. (a) SEED simulation of the radial dose deposited by secondary electrons along the track of $T=0.1-10 \mathrm{MeV}$ proton beams incident on PMMA. (b) Radial dose divided by the stopping power $S_{p}$ corresponding to each proton energy.

physically motivated DRF angular distribution. We would like to stress that when not detailed calculations are available, the isotropic angular distribution should be employed instead of the widely used Rudd and Kim formula [22].

To assess the influence in the radial dose of electronphonon and trapping due to polaronic effects (two processes associated to the condensed phase nature of the target), we compare in Fig. 5 the results of the radial dose obtained when removing in the SEED code these processes, for 0.1 and $1 \mathrm{MeV}$ protons in PMMA. As it can be seen, ignoring the contribution of phonons practically does not affect the radial dose, whereas removal of polarons leads to a radial dose that extends farther compared to the full simulation. However these changes are very small (only noticeable for the lowest proton beam energies) at distances where the radial dose has already fallen several orders of magnitude ( $r \gtrsim 5 \mathrm{~nm}$ in both cases). We have checked that the total energy deposited around the track remains unchanged when the interaction with phonons and polarons are either included or neglected.

We show in Fig. 6(a) the simulated radial doses obtained with the SEED code for protons in PMMA in the range of incident energies $0.1-10 \mathrm{MeV}$. Our results confirm that the deposited radial dose changes sizeably with proton energy for all radial distances, being the difference more than one order of magnitude for the closest distances to the proton track. This is directly related to the evolution of the magnitude of the 
SDiMFP with proton energy: The lower the proton energy, the more secondary electrons are ejected, so the more energy is deposited around the ion path. At larger distances the radial doses decrease similarly for all the proton energies, except for the lower ones, for which the radial dose sharply falls at $r \sim 10 \mathrm{~nm}$. This behavior is due to the maximum energy that can be transferred from the proton to energetic electrons: As seen in Fig. 1, the high energy tail of the SDiMFP grows with the proton energy, since more energetic electrons can be produced. Although not very numerous, these electrons carry large amounts of energy and can travel far away, then justifying the maximum extension of the radial dose, which accordingly increases with proton energy. These trends are of great relevance for the different applications, such as proton beam writing or ion beam cancer therapy: Lower proton energies produce both (i) larger amounts of energy deposited and (ii) larger concentration of these energies in shorter distances, thus increasing the efficiency and spatial resolution of the proton beam techniques. The radial dose dependence as a function of the distance $r$ from the proton track only follows an approximate $r^{-2}$ behavior for intermediate distances, with sizable deviations appearing at large distances $r$, analogously to the results obtained in similar studies performed for other targets and projectiles using different methodologies [58-60].

It is worth noticing, as depicted in Fig. 6(b), that the different radial doses practically collapse to a single curve (up to $5 \mathrm{~nm}$ ) when divided by the stopping power $S_{\mathrm{p}}$ for each proton energy [28]. This behavior can be understood when considering that the integral of the radial dose over the radial distance converges to the total energy lost by the proton per unit path length, i.e., the stopping power, which increases at the lower proton energies discussed in this work. The maximum stopping power of PMMA for protons occurs at around $0.1 \mathrm{MeV}$. First, this (almost) common curve is a useful feature for predicting radial doses when the stopping power (a well know quantity in most cases) is provided. Furthermore, it remarks (again) the role of the low energy electrons in the build up of the radial dose at short radii. As depicted in Fig. 1, the maximum of the SDiMFP always appears at the same (low) electron energy $W$ for all the incident proton energies $T$. This yields, as it has been demonstrated in previous works [14], an average kinetic energy of the secondary electrons which, in the energy range studied, is always around $50 \mathrm{eV}$, only slightly changing with the proton energy. Therefore, no matter what the energy of the impinging proton is, the radial redistribution of the energy at short distances will be always quite similar, owing to the fact that low energy electrons (the most abundant) are always emitted with similar energies (and with isotropic angular distributions, as already pointed out above). The differences in the radial dose will be more related to the amount of energy deposited (the number of electrons ejected), rather than to the extent to which this energy is transported radially. Even though, as previously discussed, the energy of the primary proton will determine the maximum extension of the radial dose, due to the role of the more energetic electrons, which are few but very effective in generating cascades of further electrons that carry the energy away from the proton track.

Our results for the radial dose around the tracks of energetic protons in PMMA are compared in Fig. 7 to previous Monte Carlo results by Udalagama et al. [17] for $0.5-3 \mathrm{MeV}$ proton beams, which correspond to conditions of practical interest in

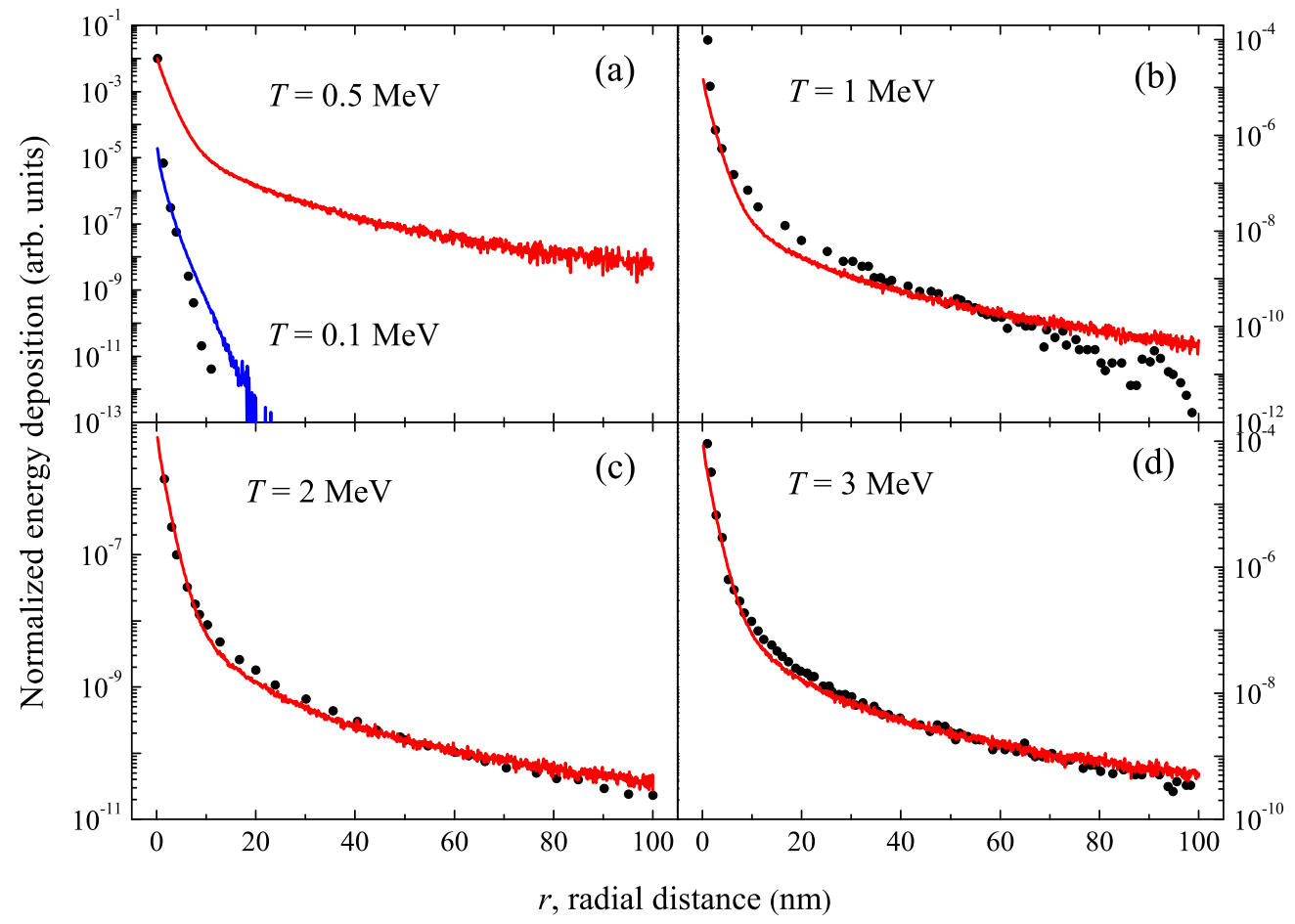

FIG. 7. Energy deposited radially by secondary electrons along the track of (a) $0.5 \mathrm{MeV}$, (b) $1.0 \mathrm{MeV}$, (c) $2.0 \mathrm{MeV}$, and (d) $3.0 \mathrm{MeV}$ proton beams incident on PMMA. Results obtained in this work with the SEED code (red curves) are compared with previous simulations (symbols) [17]. In panel (a) our simulation for $0.1 \mathrm{MeV}$ protons (blue curve) is also plotted for comparison purposes. See main text for more details. 
the proton beam writing technologies [61]. Since no units were provided in Ref. [17], we have scaled their results with ours for each proton energy to find the best match and compare the general trends obtained in both studies. It can be noticed in Fig. 7 that our simulations agree fairly well with those for the higher energies (1, 2 and $3 \mathrm{MeV})$. However, important differences are found for the case of $0.5 \mathrm{MeV}$ protons, where our curve goes to much larger radial distances, following the behavior discussed in Fig. 6(a).

The results depicted in Fig. 7 are relevant to choose the appropriate energies for proton beam writing or lithography [61], in order to avoid proximity effects that lead to degrading the resolution of these techniques. The recommended energy of $0.5 \mathrm{MeV}$ in Ref. [17] was chosen due to the compactness of the radial dose profile, justifying such election on the production of less energetic electrons for low energy proton beams, which travel to shorter distances from the incident projectile track. Although this line of reasoning is correct, as we have seen from the previous discussion, Udalagama et al. [17] only extended their analysis down to $0.5 \mathrm{MeV}$. Our results for this energy clearly disagree with these authors, but we find a similar radial dose around the proton track when going down to $0.1 \mathrm{MeV}$ [also depicted in Fig. 7(a)], which is the energy at which the maximum of the stopping power of PMMA for protons takes place [28]. Therefore, proximity effects around each proton track decrease when using lower proton energies than previously suggested.

Since our results do not consider the possible radial widening of the proton beam due to elastic scattering with the PMMA atomic nuclei, detailed simulations of the proton beam lateral spreading as it propagates through the target, both as a function of its initial energy and target depth, are required to properly evaluate proximity effects and increase resolution in proton-beam writing or lithography.

Following all the results discussed in this section, $0.1 \mathrm{MeV}$ protons present the maximum stopping power in PMMA and hence generate the maximum number of secondary electrons, so the radial dose is maximized. At the same time, at these low proton energies, the production of energetic electrons is suppressed, so the spatial concentration of energy is also maximized. Both facts lead to very intense and narrow $(\sim 10 \mathrm{~nm})$ profiles of energy deposition around each proton track, which are optimal for proton-beam writing or lithography. Besides, as low proton energies are reached at the Bragg peak by the $\sim 100 \mathrm{MeV}$ proton beams currently used in proton beam cancer therapy [31], the higher density of energy deposited around the proton tracks would lead to more severe biodamage.

\section{SUMMARY AND CONCLUSIONS}

An improved version of the event-by-event Monte Carlo code SEED (Secondary Electron Energy Deposition) has been used to describe, within a unified framework based on the dielectric response function (DRF), the production and transport of secondary electrons due to proton impact in condensed phase targets. We have considered realistic energy and angular distributions of the electrons initially generated by proton beams, as well as the subsequent incorporation of further electrons to the cascade resulting from the impact of these electrons. Besides ionization and excitation, Coulomb scattering and other energy loss channels for the moving electrons (such as polarons and phonons) have been taken into account, which become important for electrons moving at low energies through condensed-phase targets.

The cross sections for the most important electronic interaction events, namely, ionization and excitation, have been calculated within the dielectric formalism, which is a very convenient approach to treat the inelastic interactions of charged particles in condensed matter. This methodology has been applied with success to PMMA and a large number of organic and inorganic materials. The extension of the dielectric formalism to separate the excitation and ionization phenomena for arbitrary organic materials allows the calculation of the generated-electron energy and angular spectra, providing all the needed electronic cross sections to feed the simulation code SEED.

We have studied in detail the case of electron cascades generated when bombarding PMMA with proton beams, which has practical applications in proton beam writing and lithography. Using the SEED code and the implemented set of electronic cross sections, we have simulated the radial doses arising in PMMA around $0.1-10 \mathrm{MeV}$ proton tracks. The influence on radial doses due to the initial energy and angular distributions of the electrons generated by the proton beam has been analyzed. First, we have evaluated the effect on the radial energy deposition profile of using a realistic angular distribution of electrons generated by proton impact, comparing the simulated radial energy deposition with those obtained from simpler approaches often used in Monte Carlo simulations or other analytical approaches, namely distributions that are isotropic, perpendicular, forward, and backward to the proton track, as well as the widely used Rudd and Kim formula. We conclude from our simulations that an isotropic distribution produces almost identical radial energy distributions as the ones obtained from realistic angular distributions. In turn, the Rudd and Kim formula produces identical results to the perpendicular distribution, while the forward and backward distributions yield similar results, all the latter being different from the former. Therefore, we conclude that it is justified to use the very straightforward approximation of isotropic ejection of electrons generated by swift protons, since it produces results similar to the realistic angular spectrum implemented in this work. This coincidence is due to the prominent role of the low energy electrons (the most abundant) in the build up of the radial dose, which present a rather isotropic angular distribution, as our model based on the dielectric formalism reveals.

The comparison of the radial doses around 0.1-10 MeV protons in PMMA reveal a quasiuniversal behavior. Namely, the differences in the doses at short radial distances (up to $5 \mathrm{~nm}$ ) can be exclusively attributed to the different number of ejected electrons but not to their energy distribution. The radial doses normalized to the corresponding proton stopping power are very similar for short radial distances, highlighting the role of the low energy electrons on the buildup of the dose at short distances. Since the average energy of electrons produced in the situations discussed in this work is around $50 \mathrm{eV}$ [14], and it only slightly changes with the proton energy, the radial dose profile divided by the stopping power is practically universal for short radial distances. The differences 
appear for large radial distances, where the dose is due to energetic electrons. The number and maximum energy of the more energetic electrons grow with the primary proton energy, and thus the tail of the radial dose at large distances also grows with the proton energy.

Finally, our simulations are used to discuss how the resolution of proton beam writing and lithographic technologies depends on the proton beam energy, by comparing our results with previous Monte Carlo simulations [17]. Our results show that lower energy protons (i) increase the amount of dose deposited in the material (following the increase in the stopping power and the ionization cross section with decreasing energy) and (ii) transport the energy deposited to shorter distances, owing to the suppressed production of more energetic electrons. Our simulations agree with those by Udalagama et al. [17] for the higher proton energies reported, although sizable differences are found for $0.5 \mathrm{MeV}$, the lowest energy reported by these authors. We find such great levels of concentration of energy around a proton track for $0.1 \mathrm{MeV}$ protons.

We conclude that lower proton energies are recommended for proton beam based technologies requiring high energy deposition around small volumes, such as proton beam writing or lithography, among other applications. In these cases, the use of lower proton energies is more convenient, which correspond to the maximum of the stopping power and yield the highest concentrations of the radial dose (extending to $\sim 10 \mathrm{~nm}$ around each proton track).

\section{ACKNOWLEDGMENTS}

We thank I. Kyriakou (University of Ioannina) for useful discussions and P. Hoyer (University of Helsinki) for a critical reading of the manuscript. Monte Carlo simulations of secondary electron emission were performed on the KORE cluster at FBK. M.D. wishes to express warm thanks to Giovanni Garberoglio (ECT*-LISC, Trento) and Simone Taioli (ECT*-LISC, Trento) for their stimulating suggestions. Financial support was provided by the Spanish Ministerio de Economía y Competitividad and the European Regional Development Fund (Project No. FIS2014-58849-P), as well as by the Fundación Séneca (Project No. 19907/GERM/15). P.d.V. acknowledges financial support from the European Union's FP7 People: Marie-Curie Actions program within the Initial Training Network No. 608163 “ARGENT”, Advanced Radiotherapy, Generated by Exploiting Nanoprocesses and Technologies.
[1] P. Sigmund, Particle Penetration and Radiation Effects (Springer-Verlag, Berlin, Heidelberg, 2006).

[2] R. F. Egerton, Electron Energy-Loss Spectroscopy in the Electron Microscope, 3rd ed. (Springer, New York, 2011).

[3] L. Giuntini, Anal. Bioanal. Chem. 401, 785 (2011).

[4] H. Ryssel and H. Glawischning, Ion Implantation Techniques (Springer, Berlin, 1982).

[5] G. McFarland, Microprocessor Design: A Practical Guide from Design Planning to Manufacturing (McGraw-Hill Professional, New York, 2006).

[6] R. Minqin, J. A. van Kan, A. A. Bettiol, L. Daina, C. Y. Gek, B. B. Huat, H. Whitlow, T. Osipowicz, and F. Watt, Nucl. Instrum. Methods Phys. Res. B 260, 124 (2007).

[7] F. Watt, M. B. H. Breese, A. A. Bettiol, and J. A. van Kan, Mater. Today 10, 20 (2007).

[8] J. E. E. Baglin, Appl. Surf. Anal. 258, 4103 (2012).

[9] J. S. Loeffler and M. Durante, Nature Rev. Clin. Oncol. 10, 411 (2013).

[10] F. A Cucinotta and M. Durante, Lancet Oncol. 7, 431 (2006).

[11] A. A. Bettiol, Z. Mi, and F. Watt, Appl. Phys. Rev. 3, 041102 (2016).

[12] E. Surdutovich, O. I. Obolensky, E. Scifoni, I. Pshenichnov, I. Mishustin, A. V. Solov'yov, and W. Greiner, Eur. Phys. J. D 51, 63 (2009).

[13] P. de Vera, R. Garcia-Molina, I. Abril, and A. V. Solov'yov, Phys. Rev. Lett. 110, 148104 (2013).

[14] P. de Vera, I. Abril, R. Garcia-Molina, and A. V. Solov'yov, J. Phys. Conf. Ser. 438, 012015 (2013).

[15] C. P. Karger, O. Jäkel, H. Palmans, and T. Kanai, Phys. Med. Biol. 55, R193 (2010).

[16] J. A. van Kan, P. Malar, and Y. H. Wang, Appl. Surf. Sci. 310, 100 (2014).
[17] C. Udalagama, A. A. Bettiol, and F. Watt, Phys. Rev. B 80, 224107 (2009).

[18] F. A. Cucinotta, R. Katz, J. W. Wilson, and R. R. Dubey, NASA Technical Memorandum 3497, Heavy Ion TrackStructure Calculations for Radial Dose in Arbitrary Materials (1995).

[19] E. Surdutovich and A. V. Solov'yov, Eur. Phys. J. D 69, 193 (2015).

[20] H. Nikjoo, S. Uehara, and D. Emfietzoglou, Interaction of Radiation With Matter (CRC, Boca Raton, FL, 2012).

[21] M. Dapor, I. Abril, P. de Vera, and R. Garcia-Molina, Eur. Phys. J. D 69, 165 (2015).

[22] M. E. Rudd and Y. K. Kim, Rev. Mod. Phys. 64, 441 (1992).

[23] ICRU, Secondary electron spectra from charged particle interactions, Report 55 (International Commission on Radiation Units and Measurements, Bethesda, Maryland, 1996).

[24] J. Lindhard, K. Dan. Vidensk. Selsk. Mat. Fys. Medd. 28, No. 8 (1954).

[25] R. H. Ritchie, Phys. Rev. 106, 874 (1957).

[26] P. de Vera, R. Garcia-Molina, and I. Abril, Phys. Rev. Lett. 114, 018101 (2015).

[27] M. Dapor, Transport of Energetic Electrons in Solids: Computer Simulation with Applications to Materials Analysis and Characterization, 2nd ed., Vol. 257, Springer Tracts in Modern Physics (Springer, Berlin, 2017).

[28] P. de Vera, I. Abril, and R. Garcia-Molina, J. Appl. Phys. 109, 094901 (2011).

[29] All the acronyms in this work refer to the inverse mean free path, so we have used a lower case ' $i$ ' for shortening the word inverse, instead of the usual capital 'I' for inelastic.

[30] I. Abril, R. Garcia-Molina, P. de Vera, I. Kyriakou, and D. Emfietzoglou, Adv. Quant. Chem. 65, 129 (2013). 
[31] P. de Vera, R. Garcia-Molina, and I. Abril, in Nanoscale Insights into Ion-Beam Cancer Therapy, edited by A. V. Solov'yov (Springer International Publishing Switzerland, 2017), Ch. 2, p. 61 .

[32] I. Abril, R. Garcia-Molina, C. D. Denton, F. J. Pérez-Pérez, and N. R. Arista, Phys. Rev. A 58, 357 (1998).

[33] S. Heredia-Avalos, R. Garcia-Molina, J. M. Fernández-Varea, and I. Abril, Phys. Rev. A 72, 052902 (2005).

[34] N. D. Mermin, Phys. Rev. B 1, 2362 (1970).

[35] I. Abril, C. D. Denton, P. de Vera, I. Kyriakou, D. Emfietzoglou, and R. Garcia-Molina, Nucl. Instr. Meth. Phys. Res. B 268, 1763 (2010).

[36] D. J. Planes, R. Garcia-Molina, I. Abril, and N. R. Arista, J. Elect. Spectr. Rel. Phen. 82, 23 (1996).

[37] C. D. Denton, I. Abril, R. Garcia-Molina, J. C. Moreno-Marín, and S. Heredia-Avalos, Surf. Interf. Anal. 40, 1481 (2008).

[38] E. Shiles, T. Sasaki, M. Inokuti, and D. Y. Smith, Phys. Rev. B 22, 1612 (1980).

[39] J. J. Ritsko, L. J. Brillson, R. W. Bigelow, and T. J. Fabish, J. Chem. Phys. 69, 3931 (1978).

[40] B. Boudaïffa, P. Cloutier, D. Hunting, M. A. Huels, and L. Sanche, Science 287, 1658 (2000).

[41] A. Salin, J. Phys. B 2, 631 (1969).

[42] Dz. Belkic, J. Phys. B 11, 3529 (1978).

[43] Dz. Belkic, J. Math. Chem. 47, 1366 (2010).

[44] M. Dingfelder, Radiat. Protect. Dosim. 99, 23 (2002).

[45] M. Dapor, Appl. Surf. Sci. 391, 3 (2017).
[46] N. F. Mott, Proc. R. Soc. London Ser. 124, 425 (1929).

[47] M. Dapor, Electron-Beam Interactions with Solids: Applications of the Monte Carlo Method to Electron Scattering Problems, Vol. 186, Springer Tracts in Modern Physics (Springer, Berlin, 2003).

[48] A. Jablonski, F. Salvat, and C. J. Powell, J. Phys. Chem. Ref. Data 33, 409 (2004).

[49] R. Garcia-Molina, I. Abril, I. Kyriakou, and D. Emfietzoglou, Surf. Interface Anal. 49, 11 (2017).

[50] V. L. Ochkur, Sov. Phys. JETP 18, 503 (1964).

[51] J. M. Fernández-Varea, R. Mayol, D. Liljequist, and F. Salvat, J. Phys.: Cond. Matt. 5, 3593 (1993).

[52] H. Fröhlich, Adv. Phys. 3, 325 (1954).

[53] J. P. Ganachaud and A. Mokrani, Surf. Sci. 334, 329 (1995).

[54] J. Llacer and E. L. Garwin, J. Appl. Phys. 40, 2766 (1969).

[55] R. Shimizu and Z.-J. Ding, Rep. Prog. Phys. 55, 487 (1992).

[56] M. Dapor, Nucl. Instr. and Meth. B 267, 3055 (2009).

[57] F. A. Cucinotta, H. Nikjoo, and D. T. Goodhead, Radiat. Environ. Biophys. 38, 81 (1999).

[58] G. H. Olivera, P. D. Fainstein, and R. D. Rivarola, Phys. Med. Biol. 41, 1633 (1996).

[59] D. Emfietzoglou, K. Karava, G. Papamichael, and M. Moscovitch, Radiat. Protect. Dosim. 110, 871 (2004).

[60] P. de Vera, E. Surdutovich, N. J. Mason, and A. V. Solov'yov, arXiv:1703.04602v1 [physics.bio-ph] [Eur. Phys. J. D (to be published, 2017)].

[61] M. J. Madou, Manufacturing Techniques for Microfabrication and Nanotechnology, 3rd ed. (CRC Press, Boca Raton, 2011). 